\title{
POLYCYCLIC AROMATIC HYDROCARBONS AND THE EXTINCTION CURVE
}

\author{
G. Mulas ${ }^{1}$, G. Malloci ${ }^{1}$, C. Joblin ${ }^{2,3}$ and C. Cecchi-Pestellini ${ }^{1}$
}

\begin{abstract}
Aromatic carbon, in some form, has been an essential ingredient by and large in all models of the extinction curve, since the original proposal to attribute the bump at $217.5 \mathrm{~nm}$ to "astronomical graphite". This aromatic carbon is most naturally identified, in up to date models, with a population of Polycyclic Aromatic Hydrocarbons (PAHs), free and/or clustered. In all models, this PAH population accounts for the far-UV nonlinear rise in the extinction curve, contributes to the bump and possibly part of the large set of unidentified, discrete absorption features in the visible (the Diffuse Interstellar Bands). We review the current state of our understanding of the contribution of PAHs to interstellar extinction, and what constraints can be imposed on the PAH population by fitting extinction models to observations.
\end{abstract}

\section{Interstellar extinction before PAHs}

Interstellar extintion has a long, winding story. It dates as far back in time as 1774, with sir William Herschel noticing a region in the Scorpio constellation remarkably devoid of stars, which he called a "hole in the sky". The fact that such regions were a common occurrence in the Milky Way was clear in the early years of 1900, with the systematic observations of Barnard, as was stated in a textbook of the time (Clerke 1903), and it began to be hypothesised that they were not actually holes in the fabric of the sky, but instead might be "obscured" by some intervening material. For some decades, this hypothesis was the topic of some heated debates, some of which are on record in the proceedings of academic meetings of the time (Shapley \& Curtis 1921). It was only about in 1930 that firm evidence of interstellar extinction became available (Trumpler 1930).

\footnotetext{
1 Istituto Nazionale di Astrofisica - Osservatorio Astronomico di Cagliari - strada 54, località Poggio dei Pini, 09012 - Capoterra (CA)

${ }^{2}$ Université de Toulouse, UPS, CESR, 9 Av. colonel Roche, 31028 Toulouse Cedex 4, France

3 CNRS, UMR 5187, 31028 Toulouse, France
} 
Thus far, interstellar extinction was accessible to study mainly in the visible part of the spectrum, where it was known to increase linearly for decreasing wavelength, when expressed in magnitudes vs. wavenumbers (hence the name "reddening" still widely used for it), and was therefore believed to be essentially due to scattering and absorption by some form of classical dust particles. The precise composition and structure of this astronomical dust was not really constrained, as about any distribution of classical particles made of any refractory material would produce a linear extinction curve in the visible. It was only about in the 1940 decade that van de Hulst produced the first full fledged model of interstellar extinction based on well-defined dust particles (van de Hulst 1949). The only structure known at that time in this almost linear behaviour of extinction was a set of optically thin, unidentified features, called the Diffuse Interstellar Bands (DIBs). The first ones were discovered by Merrill (1934), their number has been growing steadily with the increasing sensitivity of instruments and currently exceeds four hundreds (Hobbs et al. 2010), still with no unambiguous identification despite some tentative ones. Since DIBs are the subject of some other contributions (Snow \& Desheed, Cox in this volume), we will only briefly mention them here. For a beautiful and thorough review of the early studies of interstellar extinction, see e.g. Greenberg \& Shen (1999).

The opening of the UV section of the spectrum to astronomical observations, with the advent of rocket- (Stecher 1965) and space-based (see e.g. Bless \& Savage 1970, and references therein) instruments, revealed more structure in the thus far relatively simple extinction curve. The slope of its linear behaviour changed in the near UV, and on top of it appeared two strong features: a broad Drude-like absorption "bump" peaking at about $217 \mathrm{~nm}$, followed by a non-linear additional rise in the extinction at still lower wavelengths in the far-UV. The phenomenological behaviour of the extinction curve in the visible and UV was summarised in the monumental, systematic works by Fitzpatrick \& Massa $(1986,1988,1990,2005$, $2007,2009)$. They collected the observed extinction curves for several hundreds of lines of sight, and found that in the UV (i.e. for $\lambda<270 \mathrm{~nm}$ ) they could all be parametrised satisfactorily with a relatively simple functional form.

$$
k(\lambda-V)=\frac{E(\lambda-V)}{E(B-V)}= \begin{cases}c_{1}+c_{2} x+c_{3} D\left(x, x_{0}, \gamma\right) & x \leq c_{5} \\ c_{1}+c_{2} x+c_{3} D\left(x, x_{0}, \gamma\right)+c_{4}\left(x-c_{5}\right)^{2} & x \geq c_{5}\end{cases}
$$

where $x=\lambda^{-1}$ and

$$
D\left(x, x_{0}, \gamma\right)=\frac{x^{2}}{\left(x^{2}-x_{0}^{2}\right)^{2}+x^{2} \gamma^{2}} .
$$

This functional form represents the "normalised" color excess $k(\lambda-V)$, considered as a function of inverse wavelength, with the superposition of a linear contribution, a Drude profile and a quadratic term. At longer wavelengths, $k(\lambda-V)$ is very nearly linear through the visible range, with a change of slope at around $440 \mathrm{~nm}$, and tends to zero for very long wavelengths. The extinction "bump", which is the single most intense interstellar absorption feature, was found to have an almost invariable peak position, whereas its intensity and full width at half-maximum 


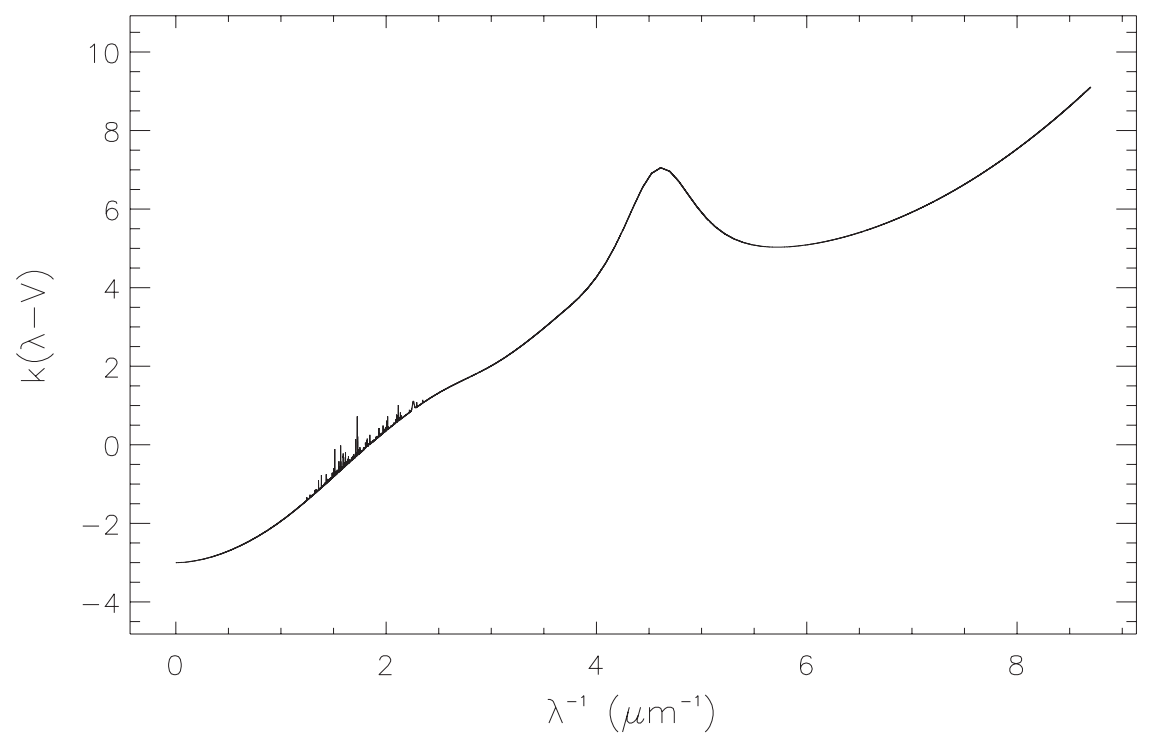

Fig. 1. Average interstellar extinction curve in the galactic plane, as given by Fitzpatrick \& Massa (2007). The definition of $k(\lambda-V)$ is given in Equation (1). The weak structure in the visible range is the set of Diffuse Interstellar Bands, taken from Hobbs et al. (2010), and arbitrarily enhanced by a large factor to make them visible on the scale of this plot.

vary substantially from one line of sight to another. The average extinction curve in the galactic plane is shown in Figure 1. The overall shape of the extinction curve in the UV suggested the presence of a large population of very small dust particles, the so called "Platt particles" after their original proposer (Platt 1955). It was then suggested, soon after the availability of the first rocket data, that such particles could actually consist of some sort of aromatic carbon (Donn 1968). Indeed, from the integrated intensity of the bump it is straightforward to obtain a robust constraint on the abundance of its carrier (see e.g. Draine 1989)

$$
\frac{n_{x}}{n_{\mathrm{H}}} f_{x}=9.3 \times 10^{-6}
$$

where $\frac{n_{x}}{n_{\mathrm{H}}}$ is the abundance of the "bump" carrier, and $f_{x}$ the oscillator strength of the transition producing it. It is apparent that even assuming a very large oscillator strength $f_{x}$ only the most abundant elements can possibly produce the bump. If one takes $f_{x} \sim 0.16$, appropriate for intensity per $\mathrm{C}$ atom of the very strong $\pi^{*} \leftarrow \pi$ resonance in graphitic materials, producing the bump observed in the average galactic extinction curve requires about $\frac{1}{4}$ of the available interstellar carbon. No other carriers could realistically produce such a strong feature compatibly with interstellar abundances. 
The above argument, together with the resemblance of the observed "bump" profile with the $\pi^{*} \leftarrow \pi$ bands in graphite, led to the development of dust models attempting to fit the entire interstellar extinction curve (Mathis et al. 1977; Lee \& Draine 1984; Draine 1987a). These models assumed power-law size distributions of spherical silicate and graphite particles, and used Mie theory, together with "optimised" optical constants (Lee \& Draine 1984; Draine 1985; Draine 1987b), obtaining a very successful fit to the observed extinction curves. One drawback was that the "optimised" optical constants did not match any precise known material, but were obtained by collecting the (then available) experimental data, with subtle ad hoc changes to make sure that they joined smoothly, that they were consistent with the Kramers-Kronig relations, and that they fitted the astronomical observations.

\section{PAHs enter the scene}

The opening of another previously unavailable spectral window, again with spacebased observations, was key to the appearance of PAHs in astronomy. The first IR observations showed a large excess of emission in the $12 \mu \mathrm{m}$ IRAS band, with respect to the others at longer wavelengths. IR spectra, furthermore, showed an ubiquitous, relatively invariant spectrum of bright emission bands at $\sim 3.3,6.2$, 7.7, 8.6, and $11.3 \mu \mathrm{m}$. Sellgren (1984) was the first to notice that especially the $3.3 \mu \mathrm{m}$ band could not be accounted for by equilibrium thermal emission of dust particles, since it would require unrealistically high temperatures. She hinted, remembering the proposed role of temperature fluctuations in the evaporation of ice mantles of dust grains (Purcell 1976; Greenberg \& Hong 1974), that these bands might be due to a population of particles with such a small thermal capacity to be transiently heated to the required high temperatures by a single UV photon. Sellgren's proposal, in hindsight, was reminiscent of the early suggestion by Platt (1955) and Donn (1968) for extinction ("Platt particles"),

Soon thereafter, two groups independently proposed that these "particles" could be free-flying Polycyclic Aromatic Hydrocarbons (PAHs) (Léger \& Puget 1984; Allamandola et al. 1985), since they appeared to fit all the required characteristics: they absorb effectively UV radiation, convert it with high efficiency to vibrational excitation, and release it as IR emission, in bands qualitatively matching the observed "Unidentified Infrared Bands" (UIRs). They are also very resistant to photodissociation (provided they are large enough), and can possibly form in C-rich outflows of evolved stars, with chemical pathways inspired from combustion chemistry. Shortly thereafter, it was also realised that since interstellar PAHs, depending on size and charge state, would have also discrete bands in the visible, this made them very plausible candidate carriers for DIBs (van der Zwet \& Allamandola 1985; Léger \& d'Hendecourt 1985).

Purely energetic considerations, based on the overall integrated emission in the UIRs, assuming unit conversion efficiency and UV absorption cross-sections (Puget \& Léger 1989; Joblin et al. 1992), led to estimate that about 15-20\% of 
the available interstellar carbon must be locked in PAHs to produce the observed IR emission fluxes. This led to the consequence that PAHs must be taken into account as a necessary ingredient of interstellar extinction models (Puget \& Léger 1989).

Indeed, from this point on, all interstellar extinction models got updated to include some sort of "astronomical PAH" component (Désert et al. 1990; Siebenmorgen \& Kruegel 1992; Li \& Greenberg 1997; Dwek et al. 1997). In particular, all models use these "astronomical PAHs" to account for the non-linear far-UV rise, very effectively decoupling it from the extinction "bump" at $217 \mathrm{~nm}$. At the time, PAHs were assumed to contribute only to the non-linear far-UV rise, while the bump was still entirely attributed to a population of classical small "astronomical graphite" particles. This was very convenient, as it provided a nice physical explanation of the observed behaviour of these two components of the extinction curve, which were known to vary independently in observations.

The assumption that "astronomical PAHs" did not contribute to the bump was due to the following reasons. The bump position is known to be very nearly invariant in all lines of sight, whereas its intensity and width vary. It is also remarkably smooth and no fine structure was detected on it so far. The (few) experimental spectra of PAHs available, almost entirely neutral ones (Joblin et al. 1992; Joblin 1992) showed $\pi^{*} \leftarrow \pi$ transitions with different positions, and with considerable structure on a wavelength scale much smaller than the bump, even when mixtures were considered, making PAHs an unattractive candidate to account for the $217 \mathrm{~nm}$ extinction feature (Draine \& Malhotra 1993; Mathis 1994).

Moreover, PAHs were believed to be largely ionised (see e.g. D'Hendecourt \& Léger 1987; Verstraete et al. 1990; Salama et al. 1995) in the diffuse interstellar medium. In their seminal model, Désert et al. (1990) assumed a mixture of ionised PAHs to have no absorption in the bump region, probably reasoning that the variation of the position of $\pi^{*} \leftarrow \pi$ transitions in different molecules would smooth them out over such a wide wavelength range to make them irrelevant.

Direct measurements of the cross-sections of PAH cations were (and still are) rather complicated, since PAH cations are very reactive species. The first experiments used the technique of matrix isolation, in which a reactive species is included as a trace component in a solid which perturbs it as little as possible (e.g. a frozen noble gas like argon or neon). Irradiation of the neutrals by far-UV photons leads to a fraction of ionised species in the matrix.

Experiments like these produced a large amount of good data on the visible spectra of PAH cations (e.g. Salama \& Allamandola 1992a, 1992b), mainly aiming at the comparison with astronomical spectra of the DIBs. For this spectral region the technique is rather effective, since the neutral PAHs studied have no features in the visible whereas the cations do. Measuring the strong $\pi^{*} \leftarrow \pi$ transitions in the bump region, however, is much more difficult, since the neutral precursors (also present in the matrix) do have strong and broad transitions there, difficult to disentangle from the features of the cations. This probably misled Lee \& Wdowiak (1993,1994a,1994b), who interpreted their experiments on irradiated 
PAH-containing glasses as showing that the $\pi^{*} \leftarrow \pi$ transitions were substantially suppressed in cations.

For all these reasons, all interstellar extinction models, for the contribution of PAHs to UV extinction, stuck with the assumption of Désert et al. (1990) and adopted an average cross-section of the neutral PAHs from which the $\pi^{*} \leftarrow \pi$ transitions were simply removed, leaving only the non-linear rise in the far UV due to the onset of $\sigma^{*} \leftarrow \sigma$ transitions (see e.g. Désert et al. 1990; Siebenmorgen \& Kruegel 1992; Li \& Greenberg 1997; Dwek et al. 1997).

Several years later, Duley \& Seahra (1998) used the discrete dipole approximation (DDA) to study the spectra of PAHs, both single and in clusters, as a function of hydrogenation and ionisation. They found that $\pi^{*} \leftarrow \pi$ transitions appeared to be almost unaffected by ionisation, in stark contrast with the previous interpretation by Lee \& Wdowiak (1993,1994a,1994b) and the assumptions of the astronomical PAH models of the time. They also found that the peak of the $\pi^{*} \leftarrow \pi$ transitions shifts systematically with dehydrogenation, and claimed that heavily dehydrogenated PAHs fit the observed position of the interstellar extinction bump. One year later, Chillier et al. (1999) measured the VUV spectrum of the perylene cation with an indirect technique, unambiguously confirming that the strong $\pi^{*} \leftarrow \pi$ transitions are almost unaffected by ionisation. In hindsight, this is hardly surprising, since, at least for large PAHs, one more or less electron does not overly perturb the $\pi$ and $\pi^{*}$ orbitals.

This awareness that all PAHs have strong $\pi^{*} \leftarrow \pi$ transitions, and the realisation that they do not leave much room in the interstellar carbon budget for a separate population of carbonaceous particles producing the bump, caused a general update of the photo-absorption cross-sections giving the contribution of PAHs to interstellar extinction models (see e.g. Weingartner \& Draine 2001; Li \& Draine 2001; Draine \& Li 2001; Draine in this volume). The far-UV part of the extinction curve, including the bump and the non-linear rise, were assumed to be completely insensitive to charge, and to include a strong $\pi^{*} \leftarrow \pi$ feature. As a consequence, models lost the flexibility to easily account for independent variation of the bump and the far-UV non-linear rise, since from now on they were assumed to be essentially due to the same population of molecules and to be largely insensitive to size and charge of the PAHs. On the other hand, the onset of absorption by PAHs became dependent on charge, as populations of PAH cations were found to begin absorbing at lower energies with respect to neutrals, reflecting experimental and theoretical results (e.g. Ruiterkamp et al. 2002,2005). PAHs became by and large the main carrier of the $217 \mathrm{~nm}$ extinction bump (see e.g. Draine in this volume).

Since all PAHs were now believed to strongly absorb in the UV, Clayton et al. (2003) searched for weak structure in the UV extinction curve, using Hubble Space Telescope observations at high spectral resolution, in the hope of being able to identify some features due to individual PAHs. This resulted in no detection, and in the placement of relatively low upper limits, meaning that no single PAH is very abundant in the diffuse ISM. 
As experimental techniques for gas-phase measurements, e.g. in cold jets, became more feasible, more and more experimental measurements of PAH spectra became available (see e.g. Bréchignac \& Pino 1999; Pino et al. 1999; Bréchignac et al. 2001; Biennier et al. 2003; Biennier 2004; Tan et al. 2005; Staicu et al. 2006; Rouillé et al. 2007; Salama et al. 2008), but mainly in the visible and near-UV, to compare them with observations of the DIBs. PAH spectra in the vacuum UV, especially for cations, being much more difficult and less promising for a direct identification, were left behind. A thorough review of the state of the art of the spectroscopy of PAHs is given in this volume by Pino et al..

\section{Systematic theoretical spectra come in}

In the same years, the steady increase in available computing power brought larger and larger PAHs within reach of quantum chemistry calculations, in particular using the Time-dependent Density Functional Theory (TD-DFT). This technique was proven to hit a sweet spot between computational cost and accuracy, and began to be routinely used both as a complement to experimental measurements, to enable the interpretation of photo-absorption spectra and the identification of bands, and to perform systematic studies on trends of the spectral behaviour of families of PAHs as a function of structure, size, ionisation state etc. (Ruiterkamp et al. 2005). However, commonly available implementations of TD-DFT are increasingly ineffective and/or inaccurate for transitions above the ionisation limit of a given molecule. This appears to be not the case for codes which implement TD-DFT in real time and represent wavefunctions by their values on a finite grid in real space, such as the open-source Octopus (Marques et al. 2003).

Malloci (2003) and Malloci et al. (2004) tested this code against the few experimental photo-absorption spectra available up to the vacuum UV, finding that the accuracy of TD-DFT calculations remained by and large the same up to high energies (a few tenths of an $\mathrm{eV}$ for band positions) as for low energies. Moreover, the computational cost, using this kind of implementation, does not change with the spectral range on which the spectrum is computed, making it particularly suitable for computing whole PAH spectra up to (and even beyond) the Lyman limit.

Having validated the approach ( $c f$. Fig. 2), Malloci and co-workers (Malloci et al. 2005, 2007a, 2007b proceeded to compute photo-absorption spectra of a large number of PAHs over the whole wavelength range relevant for interstellar extinction, and made their results publicly available on a web-based database ${ }^{1}$. This made possible a systematic study of the effect of hydrogenation and charge state. It was confirmed (Malloci et al. 2008) that dehydrogenation progressively shifts the $\pi^{*} \leftarrow \pi$ transitions bluewards, by more than $2 \mathrm{eV}$ upon complete dehydrogenation, confirming the trend previously predicted by Duley \& Seahra (1998) and by Duley \& Lazarev (2004). However, the increased accuracy of TD-DFT

\footnotetext{
${ }^{1}$ http://astrochemistry.oa-cagliari.inaf.it/database
} 


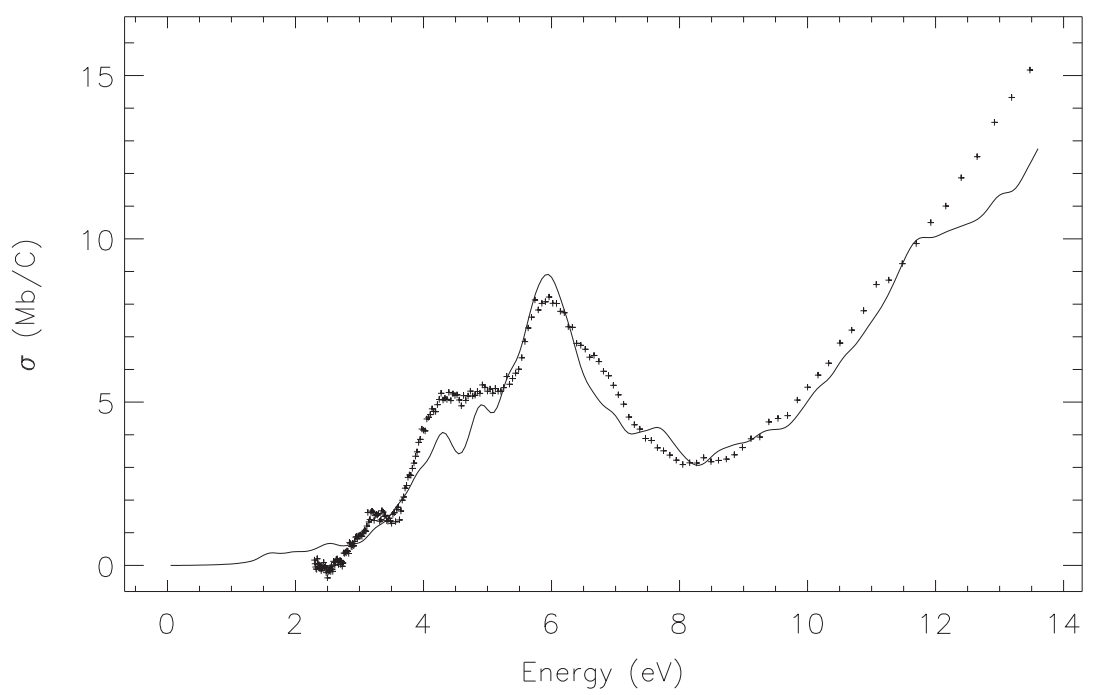

Fig. 2. Average of the photo-absorption cross-sections computed via Time-Dependent Density Functional Theory for a sample of neutral PAHs, normalised to the number of $\mathrm{C}$ atoms $\left(1 \mathrm{Mb}=10^{-18} \mathrm{~cm}^{2}=10^{-2} \AA^{2}\right)$. The theoretical curve, in continuous line, is compared with the experimental spectrum (small crosses) of a mixture of small gas-phase PAHs obtained by evaporation of a coal-pitch extract (Joblin et al. 1992).

calculations with respect to the DDA technique used by Duley \& Seahra (1998) showed that if PAHs were severely dehydrogenated their $\pi^{*} \leftarrow \pi$ absorption would fall between the "bump" and the onset of the non-linear far-UV rise, placing a strong upper limit on the overall dehydrogenation of PAHs.

As to ionisation, it was demonstrated (Cecchi-Pestellini et al. 2008) that there is indeed a relevant systematic effect: going from anions to neutrals to cations and dications it appears that while the $\pi^{*} \leftarrow \pi$ transitions (in the "bump" region) are almost unchanged, the onset of the large, broad absorption due to $\sigma^{*} \leftarrow \sigma$ transitions is progressively shifted to higher energies, significantly reducing the absorption in the wavelength range of the non-linear far-UV rise (see Fig. 3). This is important for interstellar extinction models, since it offers a physically sensible parameter which can change the observed independent variation of the "bump" and non-linear far-UV rise.

\section{Including actual PAH cross-sections in extinction models?}

The availability of a relatively large number of real computed photo-absorption spectra of PAHs made it possible to attempt, for the first time, the construction of an interstellar extinction model including a distribution of classical dust particles 


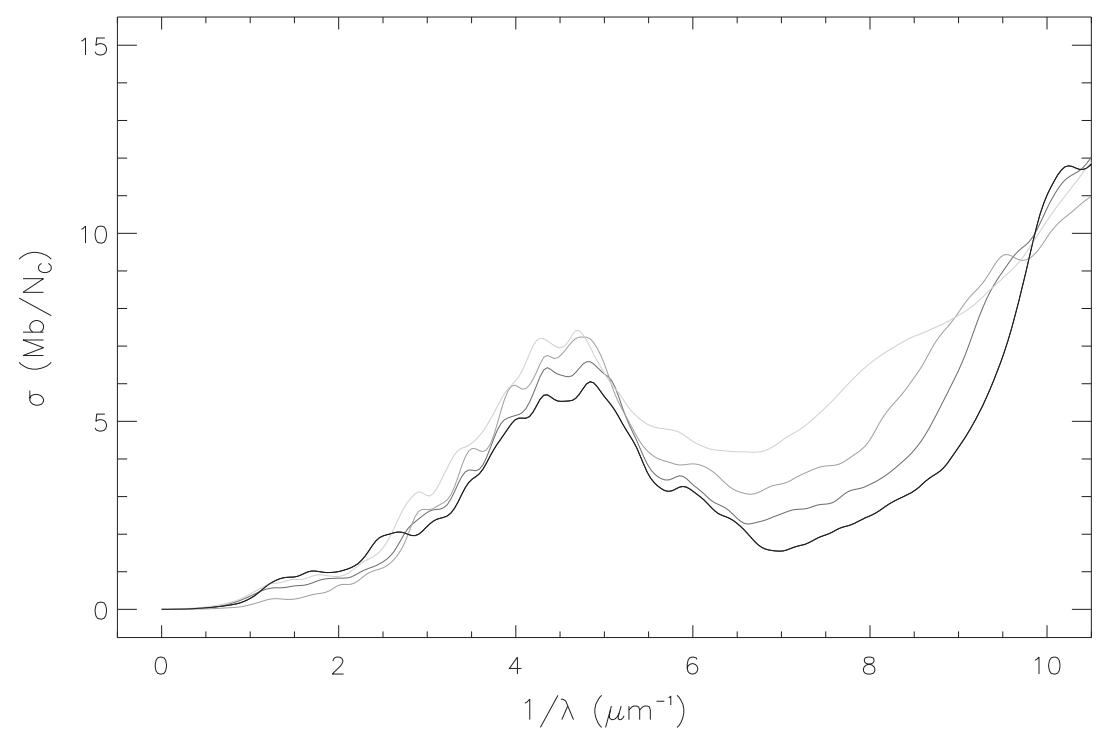

Fig. 3. Systematic variation of the average of the photo-absorption cross-sections of a sample of computed PAHs $\left(1 \mathrm{Mb}=10^{-18} \mathrm{~cm}^{2}=10^{-2} \AA^{2}\right)$, normalised to the number of $\mathrm{C}$ atoms, with charge state (anions in light gray, neutrals in medium gray, cations in dark gray, dications in black).

and a linear combination of cross-sections of a sample of PAHs in several charge states (Cecchi-Pestellini et al. 2008). This has the obvious advantage of being a more physical approach, and the obvious drawback of introducing a ludicrous number of free parameters (the column density of each species included in the lineal combination).

Comparison with observed extinction curves showed quantitatively several points. The first is that it is indeed practically possible to match the extinction curve very accurately using a mixture of even a relatively small sample of "real" PAHs. Some examples are shown in Figure 4. A few tens of species provide enough chemical diversity to very effectively wash out spectral features of individual species in the UV, resulting in a very smooth extinction curve which matches very accurately the observed one in the UV, including both the "bump" and the far-UV rise. While this would now appear obvious in hindsight, Figures 2 and 3 make it evident that not every mixture of PAH spectra results in washing out spectral structure: an actual fit on real observations was necessary to prove that obtaining a smooth bump with PAHs is not only possible but actually easy (the fit is very strongly underdetermined, see below). On the other hand, at least in some peculiar lines of sight there must be some (probably carbonaceous) component of very small (nanometer sized) particles other than PAHs, as PAHs cannot reproduce extreme cases with a strong non-linear far-UV rise and no bump. Several works showed that one feasible component with the required properties would be 


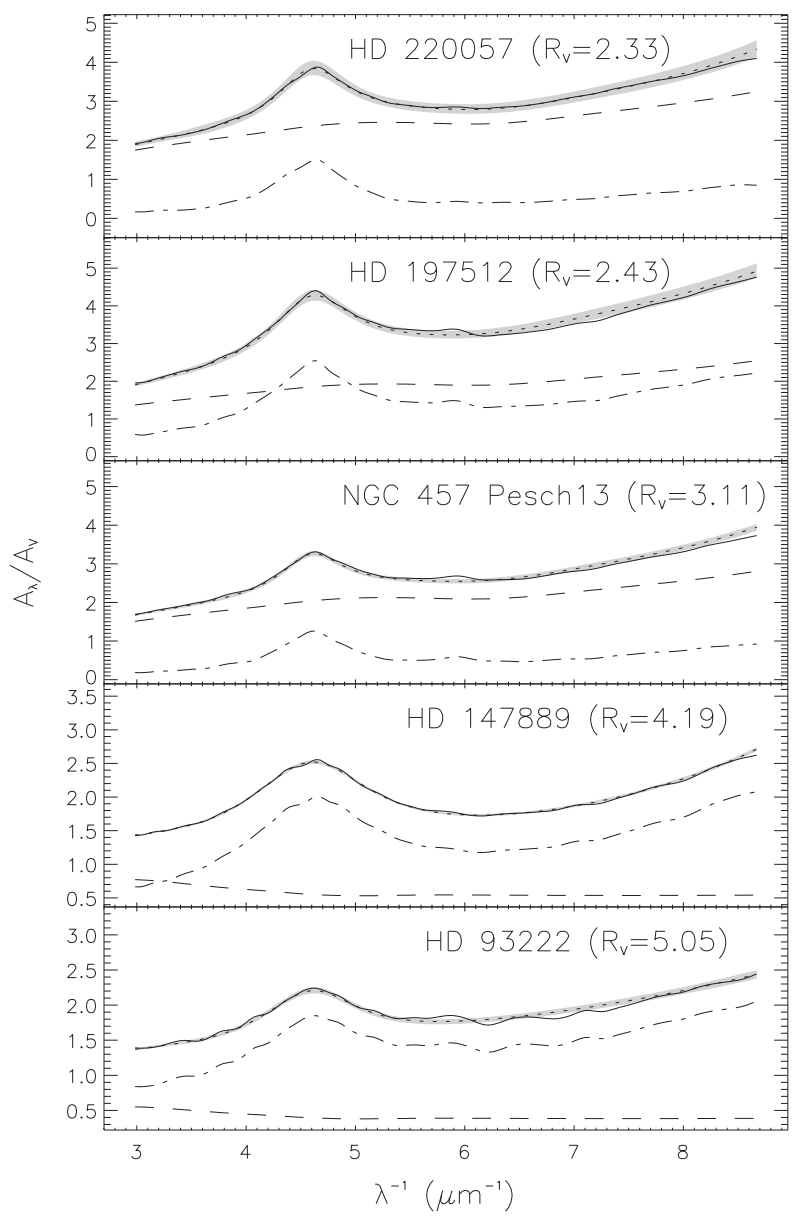

Fig. 4. Comparison between some observed extinction curves (dotted lines, Fitzpatrick \& Massa 2007), spanning a large range of values of the total to selective extinction ratio $R_{V}=\frac{A_{V}}{E_{B-V}}$, and the fits (solid continuous line) obtained by Cecchi-Pestellini et al. (2008). The dashed line shows the contribution of classical dust particles, the dash-dotted lines the contribution of PAHs.

a carbonaceous material in which carbon has an $s p^{3}$ structure, such as nanodiamonds or hyperhydrogenated PAHs (see e.g. Rai \& Rastogi 2010).

The other important point is that it was quantitatively shown that even if a fit of the extinction curve with a mixture of PAHs is severely underconstrained, meaning that there is no uniquely defined composition of PAHs required to produce a given observed interstellar extinction curve, nonetheless some collective properties of the mixture are well-determined, e.g. the charge per carbon atom, which relates to the relative intensity between the "bump" and the non-linear far-UV rise. This 


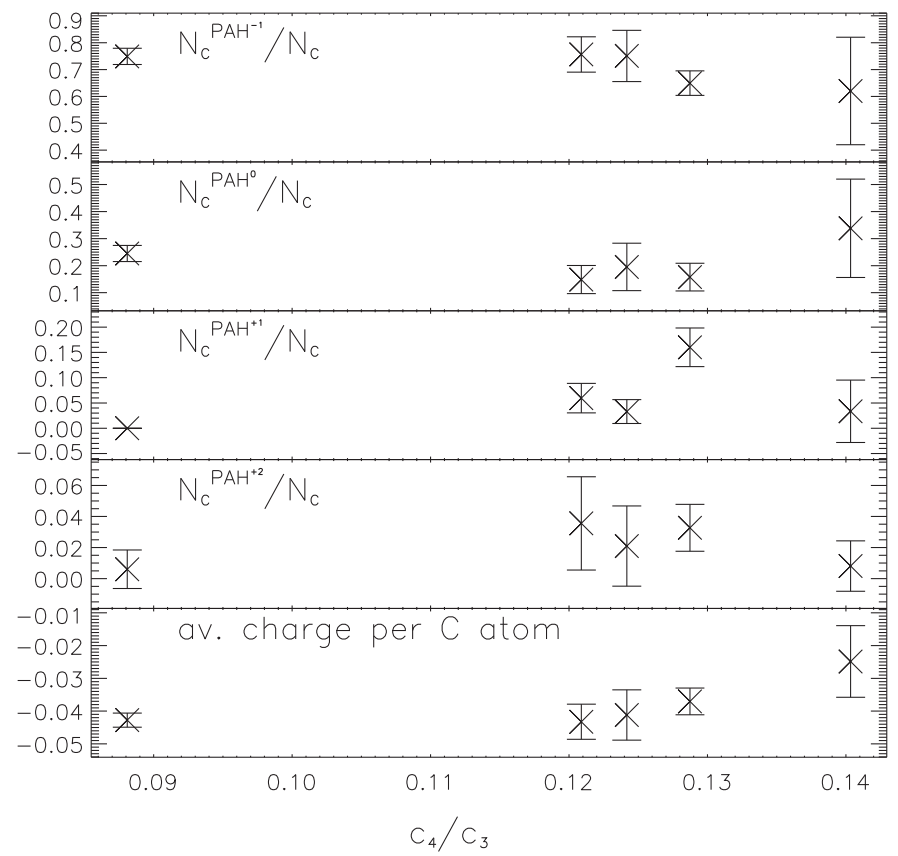

Fig. 5. Correlation between the charge (in units of electron charges) per $\mathrm{C}$ atom in PAHs and the $c_{4} / c_{3}\left(c_{4}\right.$ and $c_{3}$ are defined in Eq. (1)) ratio. From top to bottom, this is shown for $\mathrm{PAH}$ anions, neutrals, cations, dications, and the whole population. A clear trend is visible on the bottom panel.

is illustrated in Figure 5, in which the lowest panel shows that increasing ratios of the $c_{4}$ (proportional to the intensity of the non-linear far- $\mathrm{UV}$ rise) versus $c_{3}$ (proportional to the intensity of the bump) parameters in the Fitzpatrick \& Massa parametrisation (cf. Eq. (1)) correspond to an increasing value of the charge per carbon atom in PAHs. The bottom panel in Figure 5 shows quantitatively that when in an extincion curve the non-linear far-UV rise is more intense with respect to the bump, fitting it requires a mixture of PAHs with less electrons per C atom.

While such a "brute force" approach to the contribution of PAHs to interstellar extinction is probably not per se the best way for a comprehensive modelling of dust absorption and emission, it shows that the best currently available such global model (e.g. Li \& Draine 2001) lose some important information with their (necessary) simplification of PAH absorption, for example disregarding the impact of charge on far-UV extinction. As Einstein liked to say, a model should be made as simple as possible without losing significant physics, but not simpler than that. Some more work still needs to be done to achieve this result for PAHs and extinction, to find an acceptable middle ground somewhere between the nightmare of a poorly defined mixture of too many PAHs and an unrealistic single spectrum of the "average astronomical PAHs". 


\section{References}

Allamandola, L.J., Tielens, A.G.G.M., \& Barker, J.R., 1985, ApJ, 290, 25

Biennier, L., 2004, Chem. Phys. Lett., 387, 287

Biennier, L., Salama, F., Allamandola, L.J., \& Scherer, J.J., 2003, J. Chem. Phys., 118, 7863

Bless, R.C., \& Savage, B.D., 1970, IAU Symp., 36, 28

Bréchignac, P., Pino, T., \& Boudin, N., 2001, Spectrochim. Acta, 57, 745

Bréchignac, P., \& Pino, T., 1999, A\&A, 343, L49

Cecchi-Pestellini, C., Malloci, G., Mulas, G., Joblin, C., \& Williams, D.A., 2008, A\&A, 486, L25

Chillier, X.D.F., Stone, B.M., Salama, F., \& Allamandola, L.J., 1999, J. Chem. Phys., 111,449

Clayton, G.C., et al., 2003, ApJ, 592, 947

Clerke, A.M., 1903, Problems in astrophysics (London, A. \& C. Black)

Désert, F.-X., Boulanger, \& F., Puget, J.L., 1990, A\&A, 237, 215

D'Hendecourt, L.B., \& Léger, A., 1987, A\&A, 180, L9

Donn, B., 1968, ApJ, 152, 129

Draine, B.T., \& Li, A., 2001, ApJ, 551, 807

Draine, B.T., \& Malhotra, S., 1993, ApJ, 414, 632

Draine, B.T., 1989, Interstellar dust, Proc. IAU Symp., 135, ed. L.J. Allamandola \& A.G.G.M. Tielens, 313

Draine, B.T., 1987, BAAS, 19, 1077

Draine, B.T., 1987, ApJS, 64, 505

Draine, B.T., 1985, ApJS, 57, 587

Draine, B.T., \& Lee, H.M., 1984, ApJ, 285, 89

Duley, W.W., \& Lazarev, S., 2004, ApJ, 612, L33

Duley, W.W., \& Seahra, S., 1998, ApJ, 507, 874

Dwek, E., et al., 1997, ApJ, 475, 565

Ehrenfreund, P., D'Hendecourt, L., Joblin, C., \& Léger, A., 1992, A\&A, 266, 429

Fitzpatrick, E.L., \& Massa, D., 2009, ApJ, 699, 1209

Fitzpatrick, E.L., \& Massa, D., 2007, ApJ, 663, 320

Fitzpatrick, E.L., \& Massa, D., 2005, AJ, 130, 1127

Fitzpatrick, E.L., \& Massa, D., 1990, ApJS, 72, 163

Fitzpatrick, E.L., \& Massa, D., 1988, ApJ, 328, 734

Fitzpatrick, E.L., \& Massa, D., 1986, ApJ, 307, 286

Greenberg, J.M., \& Hong, S.-S., 1973, BAAS, 5, 380

Greenberg, J.M., \& Hong, S.-S., 1974, Galactic Radio Astron., 60, 155

Greenberg, J.M., \& Shen, C., 1999, Ap\&SS, 269, 33

Hobbs., L.M., York, D.G., Thorburn, J.A., et al., 2010, A New Atlas of the Diffuse Interstellar Bands: HD 183143, 65th International Symposium On Molecular Spectroscopy 
Joblin, C., Léger, A., \& Martin, P., 1992, ApJ, 393, L79

Joblin, C., 1992, Ph.D. Thesis

Lee, H.M., \& Draine, B.T., 1984, BAAS, 16, 446

Lee, W., \& Wdowiak, T.J., 1994, Molecules and Grains in Space, 312, 675

Lee, W., \& Wdowiak, T.J., 1994, The First Symposium on the Infrared Cirrus and Diffuse Interstellar Clouds, 58, 295

Lee, W., \& Wdowiak, T.J., 1993, ApJ, 410, L127

Léger, A., Verstraete, L., D'Hendecourt, L., et al., 1989, Interstellar Dust, 135, 173

Léger, A., \& d'Hendecourt, L., 1985, A\&A, 146, 81

Léger, A., \& Puget, J.-L., 1984, A\&A, 137, 5

Li, A., \& Draine, B.T., 2001, ApJ, 554, 778

Li, A., \& Greenberg, J.M., 1997, A\&A, 323, 566

Malloci, G., Mulas, G., \& Joblin, C., 2004, A\&A, 426, 105

Malloci, G., 2003, Ph.D. Thesis

Malloci, G., Joblin, C., \& Mulas, G., 2007, Chem. Phys., 332, 353

Malloci, G., Joblin, C., \& Mulas, G., 2007, A\&A, 462, 627

Malloci, G., Mulas, G., Cappellini, G., Fiorentini, V., \& Porceddu, I., 2005, A\&A, 432, 585

Malloci, G., Mulas, G., Cecchi-Pestellini, C., \& Joblin, C., 2008, A\&A, 489, 1183

Marques, M.A.L., Castro, A., Bertsch, G.F., \& Rubio, A., 2003, Computer Phys. Commun., 151, 60

Mathis, J.S., 1994, ApJ, 422, 176

Mathis, J.S., Rumpl, W., \& Nordsieck, K.H., 1977, ApJ, 217, 425

Merrill, P.W., 1934, PASP, 46, 206

Pino, T., Boudin, N., \& Bréchignac, P., 1999, J. Chem. Phys., 111, 7337

Platt, J.R., 1955, ApJ, 123, 486

Puget, J.L., \& Léger, A., 1989, ARA\&A, 27, 161

Purcell, E.M., 1976, ApJ, 206, 685

Rai, R.K., \& Rastogi, S., 2010, MNRAS, 401, 2722

Rouillé, G., Arold, M., Staicu, A., et al., 2007, J. Chem. Phys., 126, 174311

Ruiterkamp, R., Cox, N.L.J., Spaans, M., et al., 2005, A\&A, 432, 515

Ruiterkamp, R., Halasinski, T., Salama, F., et al., 2002, A\&A, 390, 1153

Salama, F., Tan, X., Biennier, L., et al., 2008, BAAS, 40, 187

Salama, F., \& Allamandola, L.J., 1992, ApJ, 395, 301

Salama, F., \& Allamandola, L.J., 1992, Nature, 358, 42

Salama, F., Joblin, C., \& Allamandola, L.J., 1995, P\&SS, 43, 1165

Sellgren, K., 1984, ApJ, 277, 623

Shapley, H., \& Curtis, H.D., 1921, Bull. Nat. Res. Council, 2, 217

Siebenmorgen, R., \& Kruegel, E., 1992, A\&A, 259, 614

Staicu, A., Krasnokutski, S., Rouillé, G., Henning, T., \& Huisken, F., 2006, J. Mol. Struct., 786, 105 
Stecher, T.P., 1965, ApJ, 142, 1683

Steglich, M., Jäger, C., Rouillé, G., et al., 2010, ApJ, 712, L16

Tan, X., Biennier, L., Cami, J., \& Salama, F., 2005, IAU Symp., 235, 70P

Trumpler, R.J., 1930, Lick Obs. Bull. XIV, 154

van de Hulst, H.C., 1957, Light scattering by small particles (Wiley)

van der Zwet, G.P., \& Allamandola, L.J., 1985, A\&A, 146, 76

Verstraete, L., Léger, A., D'Hendecourt, L., Defourneau, D., \& Dutuit, O., 1990, A\&A, 237,436

Weingartner, J.C., \& Draine, B.T., 2001, ApJ, 548, 296 